\title{
Relação entre o forame apical e o ápice radicular em dentes anteriores superiores humanos
}

Relationship between the apical foramen and the root apex in human maxillary anterior teeth Relación entre el foramen apical y el ápice de la raíz en los dientes anteriores superiores humanos Isaac de Sousa ARAújo

Manuela Maria de Sousa SILVA

Maria Natália Serafim TAVARES

Curso de Odontologia, Centro Universitário Doutor Leão Sampaio, UNILEÃO, 63040-405 Juazeiro do Norte - CE, Brasil

\section{Resumo}

Introdução: A anatomia da região mais apical do dente se reveste de bastante importância, pois é a partir dessa região que podemos definir o comprimento para execução de todas as etapas da terapia endodôntica. Objetivo: aferir, em condição ex vivo, a distância entre o forame principal e o vértice radicular de incisivos centrais, incisivos laterais e caninos superiores humanos, verificando a taxa de coincidência entre estas estruturas anatômicas, bem como a face de exteriorização do forame principal. Material e Método: 134 dentes anteriores superiores humanos, tiveram seus acessos realizados e a patência foraminal estabelecida com instrumentos tipo K \#10. Os mesmos foram divididos em três grupos, em função do grupamento dentário pertencente: incisivo central $(n=52)$, incisivo lateral $(n=46)$ e canino $(n=36)$, numerados de forma aleatória e fotografados com câmera digital acoplada ao Microscópio Digital, permitindo medir a extensão entre o forame apical e o vértice radicular. Resultados: as medidas médias e de desvio padrão dos comprimentos obtidas para cada grupo foram: 0,63 e 0,27 (incisivo central), 0,66 mm e 0,27 (incisivo lateral) e, 0,87 mm e 0,41 (canino). A taxa de coincidência forame/ápice para a amostra foi de $19,4 \%$, sendo maior para os incisivos laterais $(23,9 \%)$ e menor para os caninos $(13,9 \%)$. A face palatina foi onde ocorreu a maior frequência de exteriorização do forame principal. Conclusão: os resultados desta pesquisa apontam para uma baixa taxa de coincidência forame-ápice em dentes anteriores superiores humanos, sendo a distância entre as estruturas anatômicas e face de exteriorização extremamente variável.

Descritores: Endodontia; Anatomia; Ápice Dentário.

\section{Abstract}

Introduction: The anatomy of the apical region of the tooth is very important, since it is from this region that we can define the length for the execution of all phases of endodontic therapy. Objective: to measure, in ex vivo condition, the distance between the principal foramen and the root apex of central incisors, lateral incisors and canines human maxillary, checking the coincidence ratio between these anatomical structures, as well as the exterior aspect of the principal foramen. Material and Method: 134 human maxillary anterior teeth had their access performed and foraminal patency was established with K\#10 type instruments. They were divided into three groups, depending on the dental group: central incisor $(n=52)$, lateral incisor $(n=46)$ and canine $(n=36)$, randomly numbered and photographed with a digital camera attached to the microscope Digital, allowing to measure the extension between the apical foramen and the root apex. Results: the mean and standard deviation measurements of the lengths obtained for every group were: 0.63 and 0.27 (central incisor), $0.66 \mathrm{~mm}$ and 0.27 (lateral incisor) and, $0.87 \mathrm{~mm}$ and 0, 41 (canine). The foramen / apex coincidence ratio for the sample was $19.4 \%$, being higher for lateral incisors (23.9\%) and lower for canines (13.9\%). The palatine face was where the greatest frequency of exteriorization of the main foramen occurred. Conclusion: the results of this research indicate to a low rate of foramen-apex coincidence in human upper anterior teeth, with the distance between the anatomical structures and the exterior face being extremely variable.

Descriptors: Endodontics; Anatomy; Dental Apex.

\section{Resumen}

Introducción: la anatomía de la región más apical del diente es muy importante, ya que es a partir de esta región que podemos definir la longitud para la ejecución de todas las etapas de la terapia endodóntica. Objetivo: medir, en condiciones ex vivo, la distancia entre el agujero principal y el ápice de la raíz de los incisivos centrales, los incisivos laterales y los caninos superiores humanos, comprobando la tasa de coincidencia entre estas estructuras anatómicas, así como el aspecto exterior del agujero principal. Material y método: se realizó el acceso a 134 dientes anteriores superiores humanos y se estableció la permeabilidad foraminal con instrumentos tipo K \# 10. Se dividieron en tres grupos, según el grupo dental que perteneciera: incisivo central (n $=52$ ), incisivo lateral $(n=46)$ y canino $(n=36)$, numerados al azar y fotografiados con una cámara digital conectada al microscópio digital, que permite medir la extensión entre el agujero apical y el ápice de la raíz. Resultados: las mediciones de la media y la desviación estándar de las longitudes obtenidas para cada grupo fueron: 0.63 y 0.27 (incisivo central), $0.66 \mathrm{~mm}$ y 0.27 (incisivo lateral) y, $0.87 \mathrm{~mm}$ y 0,41 (canino). La tasa de coincidencia de foramen/ápice para la muestra fue de 19.4\%, siendo mayor para incisivos laterales $(23.9 \%)$ y menor para caninos (13.9\%). El lado palatino fue donde se produjo la mayor frecuencia de exteriorización del agujero principal. Conclusión: los resultados de esta investigación apuntan a una baja tasa de coincidencia foramen-ápice en los dientes anteriores superiores humanos, con una distancia extremadamente variable entre las estructuras anatómicas y el lado de exteriorización.

Descriptores: Endodoncia; Anatomía; Ápice del Diente.

INTRODUÇÃO

$$
\text { É imperioso à prática endodôntica o }
$$

respeito aos tecidos da região periapical, visto que o reparo orgânico está relacionado com a extensão apical dos procedimentos intracanais e, embora seja uma discussão baseada em milímetros, ela assume grande importância, visto que influencia diretamente o sucesso da terapia endodôntica, principalmente à longo prazo, como comprovado por estudos retrospectivos, nos quais melhores taxas de sucesso foram encontrados quando a obturação estava confinada à região intracanal, em detrimento dos tratamentos realizadas à nível de forame apical ou sobreobturações ${ }^{1,2}$.

Nesse sentido, a anatomia interna do canal radicular se reveste de bastante importância, principalmente quando se trata da região mais apical do dente, pois é a partir dessa região que podemos definir melhor 0 comprimento para execução de todas as etapas da terapia endodôntica ${ }^{3}$. Segundo a literatura, a constrição apical deve ser respeitada como 
limite para os procedimentos de instrumentação e obturação, e a distância entre este reparo anatômico, quando presente, e o forame apical varia de 0,5 a $1,0 \mathrm{~mm}$ para dentes de diferentes idades ${ }^{4,5}$

Clinicamente, é uma tarefa difícil, e por vezes empírica, localizar a constrição ou o forame apical, pois a estrutura geralmente visível radiograficamente é o ápice radicular. Porém, em mais de $60 \%$ dos canais radiculares, o forame principal não coincide com o ápice radicular, podendo ter em alguns casos uma distância entre o forame principal e o vértice radicular, onde suas medidas poderá variar de 0 a $3 \mathrm{~mm}^{6}$.

Sabendo que o exame radiográfico é apropriado para fins de diagnóstico e avaliação da macromorfologia da raiz, mas não é capaz de determinar a real localização do forame apical de forma consistente, devido a variações anatômicas entre os dentes e as limitações impostas pelas insuficientes duas dimensões radiográficas ${ }^{6,7}$, estudos que se predisponham a analisar a micromorfologia apical, em especial a relação forame-ápice dentário, são de grande valia à prática clínica da especialidade endodôntica.

O desvio do forame principal do ápice anatômico não pode ser facilmente detectado durante o exame radiográfico de diagnóstico, principalmente quando esta abertura ocorre na superfície bucal ou lingual da superfície radicular, ficando, assim, sobreposto pela estrutura radicular ${ }^{8}$. Assim, na observação radiográfica, os parâmetros de odontometria, instrumentação e obturação endodônticos podem parecer adequados, mas frequentemente ocorrem erros, quando a abertura foraminal está localizada em um ponto mais curto que o ápice anatômico ${ }^{9}$. Logo, a necessidade de um conhecimento completo e exato da topografia da anatomia dental tem sido reconhecida e isto a torna tão indispensável quanto o diagnóstico e o planejamento antes do início do procedimento clínico.

Assim, considerando o exposto, o objetivo desta pesquisa foi mensurar, ex vivo, a distância entre o forame principal e o vértice apical radicular de dentes anteriores superiores humanos. Além disso, esta pesquisa ainda se propôs a verificar a taxa de coincidência entre 0 forame apical e o vértice radicular e observar a localização do forame principal na amostra estudada. Destaca-se que 0 adequado aprendizado da anatomia interna do sistema de canais radiculares torna-se um marco de extrema relevância para um tratamento endodôntico de boa qualidade.
MATERIAL E MÉTODO

Trata-se de uma pesquisa laboratorial ex vivo aprovada pelo Comitê de Ética em Pesquisa do Centro Universitário Doutor Leão Sampaio - UniLeão, sob o parecer $\mathrm{n}^{\circ}$ 3.626.502.

Uma amostra de cento e trinta e quatro (134) dentes anteriores superiores humanos, com formação radicular completa, sem fraturas ou reabsorções radiculares, canal único, forames apicais principais patentes à instrumento tipo K 10 (Dentsply-Maillefer, Ballaigues, Switzerland), extraídos por razões protéticas, ortodônticas e/ou periodontais foram selecionados para o presente estudo. Os dentes foram imersos em hipoclorito de sódio a $5 \%$ (AudaxCo, São Paulo) durante 20 minutos, para facilitar a remoção manual de restos teciduais, cálculos e outras sujidades, com auxílio de curetas. A amostra foi lavada em água corrente e mantida em solução de cloreto de sódio a $0,9 \%$ até a realização dos procedimentos da pesquisa, nas dependências do laboratório de endodontia da UniLeão.

A amostra foi dividida em três grupos, em função do grupamento dentário pertencentes (incisivo central, incisivo lateral e canino), para a realização dos procedimentos iniciais da pesquisa, desenvolvidos por um único operador, previamente calibrado. Os acessos coronários foram realizados de maneira padronizada utilizando pontas diamantadas 1012 e 3083 (KG Sorensen, Barueri) acionadas em alta rotação sob abundante irrigação.

Estando a câmara pulpar inundada com solução de hipoclorito de sódio 2,5\% (Asfer, São Caetano do Sul), uma lima manual tipo K 10 (Dentsply-Maillefer, Ballaigues) foi inserida, com movimentos manuais de exploração do canal, até a visualização de sua ponta na saída do forame principal, com auxílio de uma lupa portátil com 10 vezes de aumento. A face de exteriorização foi anotada em formulário específico e para evitar a movimentação do instrumento no momento durante a realização da fotografia apical, uma pelota de algodão foi compactada em toda a extensão da câmara pulpar coronária, com o auxílio de um calcador tipo Paiva no 01 (Fava, Franco da Rocha).

$$
\mathrm{Na} \text { sequência obtiveram-se }
$$

microfotografias da porção apical, em posição ortoradial, do conjunto dente e lima, sempre posicionando a face de exteriorização do forame voltadas para a lente do Microscópio Digital Usb Zoom 500X (Inimake, Shenzhen, China). Para a manutenção da angulação e referência para calibração do programa de mensuração de distância, no momento da obtenção das 
imagens cada elemento dentário foi fixado com cera utilidade (Technew, Rio de Janeiro) sobre uma película milimetrada.

A distância $(d)$ do forame $(F)$ ao ápice dentário $(A)$ foi medida por meio de software de análise de imagens Image $\mathrm{J}$ (Oracle Coporation, Califórnia, EUA), por um avaliador calibrado e cegado quanto ao grupo dentário avaliado, pela localização precisa desses dois pontos fundamentais. $O$ ponto $F$ foi determinado exatamente sobre a ponta da lima posicionada no forame apical. A partir deste ponto traçou-se uma primeira reta em direção a superfície radicular. Na obtenção do ponto A traçou-se uma segunda reta perpendicular a primeira e tangente ao ápice radicular. Por conseguinte a distância forame-ápice correspondeu precisamente ao seguimento da primeira reta até a intersecção com a segunda reta.

Os dados coletados foram tabulados e analisados através do programa estatístico SPSS, versão 20 , e a análise descritiva dos resultados, disposta em tabelas, onde observou-se o padrão das determinações das distâncias calculadas no estudo e foi realizada em função das medidas mínima, média, máxima e desvio padrão dos comprimentos, além da taxa de coincidência forame/ápice para cada grupo amostral.

RESULTADOS

Os resultados da inspeção da anatomia apical foram baseados na análise macroscópica e microscópica de um total de 134 elementos dentários anteriores superiores humanos, sendo 52 incisivos centrais, 46 incisivos laterais e 36 caninos. A Tabela 1 apresenta a localização do forame principal nas faces da superfície radicular, obtidos pela observação da exteriorização de uma lima tipo K10 pelo forame principal.

Tabela 1. Distribuição das frequências obtidas de acordo com a orientação topográfica do forame principal nos dentes anteriores superiores humanos.

\begin{tabular}{|c|c|c|c|c|c|c|c|c|}
\hline \multirow{2}{*}{$\begin{array}{c}\text { Face de } \\
\text { exteriorização }\end{array}$} & \multicolumn{2}{|c|}{$\begin{array}{c}\text { Incisivo } \\
\text { Central }\end{array}$} & \multicolumn{2}{|c|}{$\begin{array}{c}\text { Incisivo } \\
\text { Lateral }\end{array}$} & \multicolumn{2}{|c|}{ Canino } & \multicolumn{2}{|c|}{ Total } \\
\hline & $\mathrm{N}$ & $\%$ & $\mathrm{~N}$ & $\%$ & $\mathrm{~N}$ & $\%$ & $\mathrm{~N}$ & $\%$ \\
\hline Vértice & 10 & 19,2 & 11 & 23,9 & 05 & 13,9 & 26 & 19,4 \\
\hline Distal & 10 & 19,2 & 08 & 17,4 & 09 & 25,0 & 27 & 20,1 \\
\hline Mesial & 06 & 11,5 & 08 & 17,4 & 12 & 33,3 & 26 & 19,4 \\
\hline Palatina & 17 & 32,8 & 08 & 17,4 & 05 & 13,9 & 30 & 22,5 \\
\hline Vestibular & 09 & 17,3 & 11 & 23,9 & 05 & 13,9 & 25 & 18,6 \\
\hline Total & 52 & 100,0 & 46 & 100,0 & 36 & 100,0 & 134 & 100,0 \\
\hline
\end{tabular}

Nos elementos dentários em que foi observada uma localização não coincidente com o extremo radicular, foi traçada uma linha paralela do centro do forame ao ponto mais apical do vértice radicular para mensuração desta distância. A distância média obtida para os incisivos centrais, incisivos laterais e caninos foi de 0,63, 0,66 e 0,87 mm, respectivamente (Tabela 2).
Tabela 2. Distribuição dos valores mínimos, médios, máximos e desvio padrão encontrados do forame apical ao ápice radicular em $\mathrm{mm}$, nos grupos avaliados.

\begin{tabular}{|c|c|c|c|c|c|}
\hline \multirow{2}{*}{$\begin{array}{c}\text { Grupo } \\
\text { dentário }\end{array}$} & \multirow{2}{*}{$\mathbf{N}$} & \multicolumn{4}{|c|}{ Valor observado (mm) } \\
\hline & & Mínimo & média & Máximo & $\mathrm{DP}^{*}$ \\
\hline Incisivo Central & 42 & 0,18 & 0,63 & 1,27 & 0,27 \\
\hline Incisivo Lateral & 35 & 0,26 & 0,66 & 1,54 & 0,27 \\
\hline Canino & 31 & 0,24 & 0,87 & 1,83 & 0,41 \\
\hline
\end{tabular}

DISCUSSÃO

Nessa pesquisa foi observado que $80,6 \%$ do total da amostra não apresentam uma relação de coincidência forame ápice, comprovando que a correspondências de localização entre estas estruturas anatômicas é mais casual do que regra. A literatura evidencia que há uma grande variação anatômica entre essas estruturas e quase sempre não há coincidência do forame apical com o ápice ${ }^{10-12}$.

De acordo com Borin et al. ${ }^{12}$ o exame radiográfico não deve ser utilizado como recurso definitivo para determinação do comprimento de trabalho no tratamento endodôntico, pois o ápice anatômico visível no raio-x, não é uma referência confiável para a realização dos procedimentos endodônticos.

O grupo dentário com menor taxa de coincidência ápice-forame foi o canino (13,9\%), e está em consenso com os resultados encontrado por Brito et al. $^{13}$, utilizando metodologia semelhante. No entanto Azeredo et al. $^{14}$, por meio da técnica de diafanização, observou uma taxa de coincidência de $56 \%$ em uma amostra de 103 caninos superiores. Os autores destacam a grande importância de se conhecer as variações em relação a abertura do forame apical, visto que nem sempre ele coincide com o ápice anatômico.

A alta frequência de desvio do forame apical em relação ao ápice dentário tem sido associada com envelhecimento e deposição de cemento, além do grupo dentário. Logo, em idosos há uma maior distância entre as estruturas anatômicas apicais que em pessoas jovens ${ }^{15}$. Assim como nos dentes posteriores há uma maior deposição de cemento como adaptação a cargas oclusais mais altas ${ }^{16}$.

Verificou-se também que em relação a face de exteriorização do forame principal, foi observado uma correspondência de incidência entre as faces proximais mesial ou distal $(39,5 \%)$ e faces vestibular ou palatina $(41,1 \%)$ na amostra global. Burgel e Borba ${ }^{6}$ alertam para a possibilidade de sobreposição de imagens durante a avaliação radiográfica quando da localização vestibular/palatina do forame apical e medições realizadas com 0 uso de instrumentos endodônticos.

A exteriorização lateral do forame apical é mais comum que a coincidência do forame 
com o ápice nos incisivos centrais superiores e essa característica anatômica pode ter influência na determinação do tamanho do instrumento apical inicial, principalmente nos casos em que o instrumento que melhor se ajusta ao forame apical for maior que $150 \mu \mathrm{m}$ $(\operatorname{lima} 15)^{11}$.

O valor de desvio padrão obtido nesta pesquisa demonstra uma dispersão ou distanciamento das medidas em relação à média. Assim, o valor de desvio padrão encontrado para os caninos superiores, em relação aos outros grupos dentários, indica uma maior variação de medidas em relação à média. Uma implicação clínica que se pode destacar em relação a este fato é que, apesar da média de medidas observada de $0,9 \mathrm{~mm}$, não se pode generalizar esta medida para a criação de padrão radiográfico e afirmar que o forame vai está exatamente nesta localização, devido ao valor de desvio padrão observado.

Diferente dos outros dois grupamentos dentários, com desvio padrão menores, com distâncias mais próximas da média, onde podese supor com maior possibilidade de generalização, para todo incisivo central e lateral superiores, de acordo com os resultados desta pesquisa, o forame está situado a uma distância 0,63-0,66 mm do ápice.

A localização precisa do forame apical pelo método radiográfico durante a terapia endodôntica não é uma tarefa de fácil solução ${ }^{17,18}$. Soma-se a este infortúnio o fato de que este método ainda é o mais utilizado para obtenção da odontometria, e utiliza como referência a imagem do ápice dentário, a partir de uma radiografia de diagnóstico ${ }^{19}$. Porém, os limites de instrumentação e obturação tem como referência a constrição apical, localizada a $\pm 0,5$ $\mathrm{mm}$ do forame apical, e procedimentos endodônticos realizados neste parâmetro tem uma maior taxa de sucesso a longo prazo ${ }^{20}$.

CONCLUSÃO

As distâncias médias forame-ápice foram 0,63 (IC), 0,66 mm (IL) e, 0,87 mm (C). Concluise ainda que houve uma baixa taxa de coincidência forame ápice em dentes anteriores superiores humanos e a face de exteriorização do forame se mostrou bastante variável na amostra estudada, com distribuições semelhante entre as faces proximais (mesial e distal) e faces vestibular e palatina, com maioria leve na incidência desta última.

\section{REFERÊNCIAS}

1. Basmadjian-Charles $C L$, Farge $P$, Bourgeois $\mathrm{DM}$, Lebrun T. Factors influencing the long-term results of endodontic treatment: a review of the literature. Int Dent J. 2002;52(2):81-6.

2. $\mathrm{Ng} \mathrm{YL,} \mathrm{Mann} \mathrm{V,} \mathrm{Rahbaran} \mathrm{S,} \mathrm{Lewsey} \mathrm{J,}$ Gulabivala K. Outcome of primary root canal treatment: systematic review of the literaturepart 1. Effects of study characteristics on probability of success. Int Endod $\mathrm{J}$. 2007;40(12):921-39.

3. Silva MHC, Oliveira PY, Lima CO, Lacerda MFLS, Girelli CFM, Avelar RAD, Brandão RGP. Importance of location of root channels during endodontic treatment. Braz $\mathrm{J}$ Health Rev. 2019;2(1):154-61.

4. Ricucci D, Langeland K. Apical limit of root canal instrumentation and obturation, part 2. A histological study. Int Endod J. 1998;31(6):394409.

5. Wu MK, Wesselink PR, Walton RE. Apical terminus location of root canal treatment procedures. Oral Surg Oral Med Oral Pathol Oral Radiol Endod. 2000;89(1):99-103.

6. Burgel MO, Borba MG. SEM analysis of apical anatomy of mandibular premolars. RFO UPF. 2011;16(1):49-53.

7. Zani M, Ribeiro FC, Azeredo RA, Barros MGN, Schneider CM, Barroso JM. In vitro analysis of the canal treated teeth in jaws CT, operating microscope and photographs. Braz J Health Res. 2010;12(3):11-6.

8. Mancilha FAB, Vance R, Habitante SM, Simões S. Comparative study of internal anatomy of the anomalous teeth between radiographic techniques and clearing method. SOTAU Rev Virtual Odontol. 2008;5:22-9.

9. Burch JG, Hulen S. The relationship of the apical foramen to the anatomic apex of the tooth root. Oral Surg Oral Med Oral Pathol. 1972;34(2):262-68.

10. Soares JA, Silveira FF, Nunes E, Jham B, Borges EF. In vitro analysis of the distance from the main foramen to the radiographic vertex of the anterior teeth. Arq Odontol. 2005;41(3): 215-25.

11. Souza RA, Dantas JCP, Colombo S, Lago M, Figueiredo JAP, Pécora JD. Location of the apical foramen and its relationship with foraminal file size. Dent Press Endod. 2011;1:64-8.

12. Borin AC, Pereira KFS, Verardo LBJ, Schweich LC, Arashiro FN, Tomazinho LF. Distance from apex to foramen and the correlation with radiographic odontometry method. Rev UNINGÁ. 2016;47(1):45-9.

13. Brito LF, Nogueira SJS, Ferreira CM, Gomes FA, Sousa BC. Prevalence of major apical foramen mismatching the root apex in root canals of human permanent teet. RSBO. 2016;13(3):188-93.

14. Azeredo RA, Trindade FZ, Rédua RB, De Paula VGG, Pimenta VM, Regiani LR, Lacerda LM, 
De Martin G. Lateral upper incisor radicular canal system study, using macroscopic cuts and clarifying technique. UFES Rev Odontol. 2005;7(1):55-62.

15. Martos J, Ferrer-Luque CM, GonzálezRodríguez MP, Castro LA. Topographical evaluation of the major apical foramen in permanent human teeth. Int Endod J. 2008;42(4):329-34.

16. Alothmani OS, Chandler NP, Friedlander LT. The anatomy of the root apex: A review and clinical considerations in endodontics. Saudi Endod J. 2013;3(1):1.

17. Assumpção TS, Bramante CM, Moraes IG, Garcia RB, Bernardileni N. Avaliação de Foramina Acessórios com o Uso do Microscópio Clínico e Electrónico de Varredura. Rev Port Estomatol Med Dent Cir Maxilofac. 2009;50(4):215-19.

18. Salonski CC, Costa EM, Lopes HL, Deonizio MD, Westphalen VPD, Neto UXS, Fariniuk LF. Evaluation of apical foramen in canine human teeth. RSBO. 2004;1(1):13-6.

19. Leal PM, Gomes RTMC. Comparative analysis of different odontometry methods. Arch Health Invest. 2019;8(2):85-90.

20.Souza RA, Dantas JCP, Colombo S, Lago M, Figueiredo JAP, Pécora JD. Relationship between the apical limit of root canal filling and repair. Endodontic Practice. 2018:12-23.

\section{CONFLITO DE INTERESSES}

Os autores declaram não haver conflitos de interesse

\section{AUTOR PARA CORRESPONDÊNCIA}

\section{Isaac de Sousa Araujo}

Rua Lourdes Victória, 1037, Cidade Universitária, 63048-240, Juazeiro do Norte - CE, Brasil

E-mail: isaacaraujo@leaosampaio.edu.br

Submetido em 14/03/2020

Aceito em 23/10/2020 